\title{
The equation $x+y=1$ in finitely generated groups
}

\author{
by
}

\author{
F. Beukers (Utrecht) and H. P. Schlickewei (Ulm)
}

1. Introduction. Let $H$ be a finitely generated subgroup of rank $r$ in $\left(\mathbb{C}^{*}\right)^{2}$. Denote by $G$ the $\mathbb{Q}$-closure of $H$, i.e. the subgroup of $\left(\mathbb{C}^{*}\right)^{2}$ consisting of all pairs $\mathbf{a}=\left(a_{1}, a_{2}\right) \in\left(\mathbb{C}^{*}\right)^{2}$ such that $\mathbf{a}^{N}=\left(a_{1}^{N}, a_{2}^{N}\right) \in H$ for some $N \in \mathbb{N}$. We are interested in an upper bound for the number of solutions $(x, y) \in G$ of the equation

$$
x+y=1 .
$$

A special case of (1) is obtained if we restrict $x$ and $y$ to the group of so-called $S$-units in an algebraic number field $K$. Here $S$ is assumed to be a finite set of places of $K$ including all infinite ones. Supposing that $d=[K: \mathbb{Q}], s=\# S$ and letting $a, b \in K^{*}$ be fixed, J. H. Evertse [3, Theorem 1] showed that

$$
a x+b y=1
$$

has not more than $3 \cdot 7^{d+2 s}$ solutions. Since $s \geq d / 2$ this implies that (2) has at most $3 \cdot 7^{4 s}$ solutions. We can apply this result to equation (1). However, the estimate will depend on the degree of the field containing $H$, and on $s$, the number of places for which the elements of $H$ have non-trivial valuation. Note that for fixed $r$ the number $s$ may have arbitrarily large values.

We shall be interested in bounds which depend only on $r$. The first such uniform result for a general subgroup $G$ of $\left(\mathbb{C}^{*}\right)^{2}$ was given in [5]. There the bound $2^{2^{26}+36 r^{2}}$ was derived for the number of solutions of equation (1). This was improved in [6] to $2^{13 r+63} r^{r}$.

In this paper we obtain

THEOREM 1.1. Let $G$ be the $\mathbb{Q}$-closure of a finitely generated subgroup of $\left(\mathbb{C}^{*}\right)^{2}$ of rank $r$. Then the equation

$$
x+y=1, \quad(x, y) \in G,
$$

has not more than $2^{8 r+8}$ solutions.

Note that this bound, apart from the numerical constants, has the same shape as Evertse's upper bound. 
It is well known that a particular application of Theorem 1.1 deals with the multiplicity of binary recurrences. Let $\left\{u_{m}\right\}_{m \in \mathbb{Z}}$ be a sequence of complex numbers satisfying the recurrence relation

$$
u_{m+2}=\nu_{1} u_{m+1}+\nu_{0} u_{m}
$$

with $\nu_{0}, \nu_{1} \in \mathbb{C}, \nu_{0} \neq 0$. Suppose that we have initial values $\left(u_{0}, u_{1}\right) \neq(0,0)$. Write $f(z)=z^{2}-\nu_{1} z-\nu_{0}$. Let $\alpha, \beta$ be its zeros. Note that $\nu_{0} \neq 0$ implies $\alpha, \beta \neq 0$. Let us assume that $\alpha \neq \beta$. Then there exist $a, b \in \mathbb{C}$ such that

$$
u_{m}=a \alpha^{m}+b \beta^{m} .
$$

Given $c \in \mathbb{C}$ we are interested in the number of solutions $m \in \mathbb{Z}$ of $u_{m}=c$. Note that the cases $a, b$ or $c$ equal to zero are uninteresting since they have either at most one solution or infinitely many trivial ones. So we assume they are non-zero. Divide on both sides by $c$, and from now on we shall be interested in the equation

$$
\lambda \alpha^{x}+\mu \beta^{x}=1 \quad \text { in } x \in \mathbb{Z},
$$

where $\lambda \mu \alpha \beta \neq 0$. We shall also assume that $\alpha, \beta$ are not both roots of unity.

As a fine point we add that if $\alpha, \beta$ are roots of unity, then the set

$$
\left\{\left(\alpha^{x}, \beta^{x}\right): x \text { solution of }(3)\right\}
$$

consists of at most two elements. This is a consequence of the fact that there exist precisely two triangles in the complex plane two of whose sides have lengths $|\lambda|,|\mu|$, whose third side is the segment $[0,1]$ and such that the side of length $|\lambda|$ ends in 0 .

Straightforward application of Theorem 1.1 with the group $H$ generated by $(\lambda, \mu)$ and $(\alpha, \beta)$ shows that (3) has not more than $2^{24}$ solutions. However, one can do much better:

THEOREM 1.2. Under the assumptions just mentioned the equation

$$
\lambda \alpha^{x}+\mu \beta^{x}=1 \quad \text { in } x \in \mathbb{Z}
$$

has at most 61 solutions.

As a curiosity we mention that the equation with the largest number of solutions known is

$$
\frac{\theta_{2}-\theta_{3}}{\theta_{2}-\theta_{1}}\left(\frac{\theta_{1}}{\theta_{3}}\right)^{x}+\frac{\theta_{1}-\theta_{3}}{\theta_{1}-\theta_{2}}\left(\frac{\theta_{2}}{\theta_{3}}\right)^{x}=1
$$

where the $\theta_{i}$ are the zeros of $X^{3}-2 X^{2}+4 X-4$. The solutions are $x=$ $0,1,4,6,13,52$. It would be interesting to have examples with more than 6 solutions, if they exist.

The first result in the situation of Theorem 1.2 with a universal bound was derived in [4] with the bound $2^{2^{23}}$. 
The improvements we give in the current paper in comparison with [4][6] depend upon two ingredients. First we use an explicit version of Thue's method via hypergeometric polynomials as given in [1], whereas the previous papers are based on a quantitative version of Roth's Theorem. To get bounds that do not depend upon degrees of number fields involved, previously a result from [7] was used on lower bounds for heights of solutions of equations. Here we apply the strongly improved bound given in Corollary 2.4 of [2].

2. Lemmas on algebraic numbers. First we fix our notations concerning heights. Let $K$ be an algebraic number field of degree $d$ over $\mathbb{Q}$. For any valuation $v$ we write $d_{v}=\left[K_{v}: \mathbb{Q}_{v}\right]$, where $K_{v}, \mathbb{Q}_{v}$ are the completions of $K, \mathbb{Q}$ with respect to $v$. For archimedean $v$ we normalise the valuation by $|x|_{v}=|x|^{d_{v} / d}$ where $|\cdot|$ is the ordinary complex absolute value. When $v$ is non-archimedean we take $|p|_{v}=p^{-d_{v} / d}$ where $p$ is the unique rational prime such that $|p|_{v}<1$. The height of an algebraic number $\alpha \in K^{*}$ is defined by

$$
H(\alpha)=\prod_{v} \max \left(1,|x|_{v}\right) .
$$

Because of our normalisation $H(\alpha)$ does not depend on the choice of the field $K$ in which $\alpha$ is contained. More generally, for any $(n+1)$-tuple $\left(x_{0}, x_{1}, \ldots, x_{n}\right) \in K^{n}$ with not all $x_{i}$ zero we define

$$
H\left(x_{0}, \ldots, x_{n}\right)=\prod_{v} \max \left(\left|x_{0}\right|_{v}, \ldots,\left|x_{n}\right|_{v}\right) .
$$

Note that by the product formula we have $H\left(\lambda x_{0}, \ldots, \lambda x_{n}\right)=H\left(x_{0}, \ldots, x_{n}\right)$ for any $\lambda \in K^{*}$, so we can view this height as a height on the $K$-rational points of the projective space $\mathbb{P}^{n}$. In particular, we have $H(\alpha)=H(1, \alpha)$.

We start with an easy lemma.

Lemma 2.1. Let $a, a^{\prime}, b, b^{\prime}, A, B \in \overline{\mathbb{Q}}^{*}$ and $c, c^{\prime} \in \overline{\mathbb{Q}}$ be such that $a b^{\prime} \neq a^{\prime} b$ and

$$
a A+b B=c, \quad a^{\prime} A+b^{\prime} B=c^{\prime} .
$$

Then $H(A, B, 1) \leq 2 H(a, b, c) H\left(a^{\prime}, b^{\prime}, c^{\prime}\right)$.

Proof. Fix a number field $K$ in which all numbers involved are contained. For each infinite valuation $v$ let $r_{v}=2^{d_{v} / d}$ and let $r_{v}=1$ if $v$ is finite. Notice that $\prod_{v} r_{v}=2$.

One easily finds that

$$
A=\frac{b c^{\prime}-b^{\prime} c}{\Delta}, \quad B=\frac{a^{\prime} c-a c^{\prime}}{\Delta}
$$


where $\Delta=a^{\prime} b-a b^{\prime}$. Hence

$$
\begin{aligned}
H(A, B, 1) & =H\left(b c^{\prime}-b^{\prime} c, a^{\prime} c-a c^{\prime}, b a^{\prime}-a b^{\prime}\right) \\
& =\prod_{v} \max \left(\left|b c^{\prime}-b^{\prime} c\right|_{v},\left|a^{\prime} c-a c^{\prime}\right|_{v},\left|b a^{\prime}-a b^{\prime}\right|_{v}\right) \\
& \leq \prod_{v} r_{v} \max \left(|a|_{v},|b|_{v},|c|_{v}\right) \max \left(\left|a^{\prime}\right|_{v},\left|b^{\prime}\right|_{v},\left|c^{\prime}\right|_{v}\right) \\
& =2 H(a, b, c) H\left(a^{\prime}, b^{\prime}, c^{\prime}\right) .
\end{aligned}
$$

As a corollary we get

Corollary 2.2. Let $a, b, A, B \in \overline{\mathbb{Q}}^{*}$ be such that $a \neq b$ and

$$
A+B=1, \quad a A+b B=1 .
$$

Then $H(A, B, 1) \leq 2 H(a, b, 1)$.

The next lemma follows from an explicit version of Thue's method using hypergeometric polynomials.

Lemma 2.3. Let $a, b, A, B \in \overline{\mathbb{Q}}^{*}$ and $\varrho \in \mathbb{N}$ be such that

$$
A+B=1, \quad a A^{2 \varrho}+b B^{2 \varrho}=1 .
$$

Then $H(A, B, 1) \leq 2^{1 / \varrho} c H(a, b, 1)^{1 / \varrho}$, where $c=6 \sqrt{3}$.

Pr o of. We infer from Lemma 6 of [1] that there exist three polynomials $P_{\varrho}, Q_{\varrho}, R_{\varrho}$ of degree $\leq \varrho$ such that

$$
\begin{gathered}
z^{2} \varrho P_{\varrho}(z)+(1-z)^{2} \varrho Q_{\varrho}(z)=R_{\varrho}(z), \quad \forall z \in \mathbb{C}, \\
b P_{\varrho}(A) \neq a Q_{\varrho}(A), \\
H\left(P_{\varrho}(A), Q_{\varrho}(A), R_{\varrho}(A)\right) \leq(6 \sqrt{3})^{\varrho} H(A)^{\varrho} .
\end{gathered}
$$

Substitute $z=A$ in the polynomial identity. Application of the previous lemma with $A^{2 \varrho}, B^{2 \varrho}$ instead of $A, B$ and $c=1, a^{\prime}=P_{\varrho}(A), b^{\prime}=$ $Q_{\varrho}(A), c^{\prime}=R_{\varrho}(A)$ yields

$$
\begin{aligned}
H(A, B, 1)^{2 \varrho} & \leq 2 H(a, b, 1) H\left(P_{\varrho}(A), Q_{\varrho}(A), R_{\varrho}(A)\right) \\
& \leq 2 c^{\varrho} H(a, b, 1) H(A)^{\varrho} \leq 2 c^{\varrho} H(a, b, 1) H(A, B, 1)^{\varrho} .
\end{aligned}
$$

Divide on both sides by $H(A, B, 1)^{\varrho}$ and take $\varrho$ th roots to obtain our lemma.

The following lemma is due to an improvement of [7] by Corollary 2.4 in $[2]$.

Lemma 2.4. Let $\lambda, \mu \in \overline{\mathbb{Q}}^{*}$ and suppose that $\lambda+\mu=1$. Let $\left(p_{i}, q_{i}\right), i=$ 1,2 , be two solutions in $\overline{\mathbb{Q}}$ of $\lambda p+\mu q=1$ such that the pairs $\left(p_{1}, q_{1}\right),\left(p_{2}, q_{2}\right)$ and $(1,1)$ are all distinct. Then

$$
H\left(p_{1}, q_{1}, 1\right) H\left(p_{2}, q_{2}, 1\right) \geq 1.0942711 \ldots
$$


By application of this lemma with $\lambda=x_{0}, \mu=y_{0}$ and $p_{i}=x_{i} / x_{0}, q_{i}=$ $y_{i} / y_{0}$ we obtain

COROllary 2.5. Let $\left(x_{0}, y_{0}\right),\left(x_{1}, y_{1}\right),\left(x_{2}, y_{2}\right)$ be three distinct solutions of $x+y=1$ in $x, y \in \overline{\mathbb{Q}}^{*}$. Then

$$
\max _{i=1,2}\left(\max \left(H\left(x_{i} / x_{0}\right), H\left(y_{i} / y_{0}\right)\right)\right) \geq 1.022777 \ldots
$$

3. Normed vector spaces. Let $m \in \mathbb{N}$. For any subgroup $H \subset\left(\overline{\mathbb{Q}}^{*}\right)^{m}$ we let the $\mathbb{Q}$-closure of $H$ be the set of all $\mathbf{a} \in\left(\overline{\mathbb{Q}}^{*}\right)^{m}$ such that $\mathbf{a}^{N} \in H$ for some $N \in \mathbb{N}$. Let $G$ be the $\mathbb{Q}$-closure of a finitely generated subgroup of $\left(\overline{\mathbb{Q}}^{*}\right)^{m}$ of rank $r$. Let $T$ be the torsion subgroup of $G$. Then $G / T=G \otimes_{\mathbb{Z}} \mathbb{Q}$ has the natural structure of a $\mathbb{Q}$-vector space of dimension $r$. Consider the logarithmic height function $h(x)=\log H(x)$. The function

$$
\left\|\left(x_{1}, \ldots, x_{m}\right)\right\|=\max _{i=1, \ldots, m} h\left(x_{i}\right)
$$

provides a natural norm on $G \otimes_{\mathbb{Z}} \mathbb{Q}$ as $\mathbb{Q}$-vector space. By continuity we can extend this norm to the real vector space $V_{G}=G \otimes_{\mathbb{Z}} \mathbb{R}$.

Lemma 3.1. The (semi)-norm $\|\cdot\|$ is positive definite on $V_{G}$.

Pro of. Let us write down the semi-norm $\|\cdot\|$ in an explicit way. Suppose the $\mathbb{Q}$-generators of $G$ are given by

$$
\mathbf{a}_{i}=\left(a_{i 1}, \ldots, a_{i m}\right), \quad i=1, \ldots, r .
$$

Any element of $G$ can be written, modulo roots of unity, in the form $\mathbf{x}=$ $\left(x_{1}, \ldots, x_{m}\right)=\prod_{i=1}^{r}\left(a_{i 1}, \ldots, a_{i m}\right)^{e_{i}}$ for some $e_{i} \in \mathbb{Q}$. Hence, using $h(a)=$ $(1 / 2) \sum_{v}\left|\log \left(|a|_{v}\right)\right|$

$$
\|\mathbf{x}\|=\max _{j=1, \ldots, m} h\left(\prod_{i=1}^{r} a_{i j}^{e_{i}}\right)=\max _{j=1, \ldots, m}(1 / 2) \sum_{v}\left|\sum_{i=1}^{r} e_{i} \log \left(\left|a_{i j}\right|_{v}\right)\right| .
$$

Extending $\|\cdot\|$ to the reals is now straightforward, simply extend $e_{i}$ to $\mathbb{R}$. We also remark that if we take the $e_{i}$ integral, the components of $\mathbf{x}$ all lie in the same number field, hence the non-trivial elements of the group generated (over $\mathbb{Z}$ ) by the $\mathbf{a}_{i}$ have a norm uniformly bounded below by a positive constant, $\gamma$, say.

We now prove positive definiteness of $\|\cdot\|$. Suppose there exists $\mathbf{y} \in V_{G}$, non-zero, such that $\|\mathbf{y}\|=0$. This implies that there exist $e_{i} \in \mathbb{R}$, not all zero, such that $\left|\sum_{i=1}^{r} e_{i} \log \left(\left|a_{i j}\right|_{v}\right)\right|=0$ for all valuations $v$ and all $j$. Using Dirichlet's box principle we can then show that for any $\varepsilon>0$ there exist integers $m_{i}$, not all zero, such that $\left|\sum_{i=1}^{r} m_{i} \log \left(\left|a_{i j}\right|_{v}\right)\right|<\varepsilon$ for all $v$ and $j$. This contradicts the existence of the uniform lower bound $\gamma$. Hence $\|\mathbf{y}\|=0$ implies that $e_{i}=0$ for all $i$, as desired. 
From now on we suppose that $G \subset\left(\overline{\mathbb{Q}}^{*}\right)^{2}$. We want to bound the number of solutions of the equation

$$
x+y=1, \quad(x, y) \in G .
$$

Consider the natural projection $p: G \rightarrow V_{G}$.

LEMMA 3.2. Let $\left(x_{0}, y_{0}\right),\left(x_{1}, y_{1}\right),\left(x_{2}, y_{2}\right)$ be three distinct solutions of $(\mathrm{M})$. Then their images under $p$ cannot be all equal.

Proof. If all three images were the same then $x_{i} / x_{0}$ and $y_{i} / y_{0}$ would be roots of unity for $i=1,2$. But this is impossible in view of Corollary 2.5.

Let $\mathcal{M}$ be the image under $p$ of the solution set of $(\mathrm{M})$. Then the number of solutions to $(\mathrm{M})$ is bounded by $2(\# \mathcal{M})$.

We now restate the lemmas of the previous section in terms of the set $\mathcal{M} \subset V_{G}$. In the derivations we use the fact that $\max (H(a), H(b)) \leq$ $H(a, b, 1) \leq \max (H(a), H(b))^{2}$.

Corollary 2.2 becomes

Lemma 3.3. Let $\mathbf{w}_{1}, \mathbf{w}_{2}$ be distinct points of $\mathcal{M}$. Then

$$
\left\|\mathbf{w}_{1}\right\| \leq \log 2+2\left\|\mathbf{w}_{2}-\mathbf{w}_{1}\right\| .
$$

Lemma 2.3 becomes

Lemma 3.4. Let $\mathbf{w}_{1}, \mathbf{w}_{2}$ be distinct points of $\mathcal{M}$ and $\varrho \in \mathbb{N}$. Then

$$
\left\|\mathbf{w}_{1}\right\| \leq \log c+\frac{1}{\varrho}\left(\log 2+2\left\|\mathbf{w}_{2}-2 \varrho \mathbf{w}_{1}\right\|\right) .
$$

Corollary 2.5 becomes

Lemma 3.5. Let $\mathbf{w}_{0}, \mathbf{w}_{1}, \mathbf{w}_{2}$ be distinct points of $\mathcal{M}$. Then

$$
\max \left(\left\|\mathbf{w}_{1}-\mathbf{w}_{0}\right\|,\left\|\mathbf{w}_{2}-\mathbf{w}_{0}\right\|\right) \geq 0.022522 \ldots
$$

It will turn out that the cardinality of any set satisfying the inequalities in the above three lemmas can be bounded in terms of the dimension of $V_{G}$.

We need some additional lemmas on coverings of convex bodies. The first is straightforward.

LEMma 3.6. Let $V$ be an m-dimensional normed real vector space with norm $\|\cdot\|$. Let $R>\delta>0$. Consider the ball $B$ of radius $R$ around the origin and suppose it contains a set $U$ such that $\left\|\mathbf{u}_{1}-\mathbf{u}_{2}\right\| \geq \delta$ for any two distinct $\mathbf{u}_{1}, \mathbf{u}_{2} \in U$. Then $\# U \leq(1+2 R / \delta)^{m}$.

Proof. Let $V_{0}$ be the volume of the unit ball $\{\mathbf{x}:\|\mathbf{x}\|<1\}$. Around any point $\mathbf{u} \in U$ we consider the open ball $B_{u}=\{\mathbf{x}:\|\mathbf{x}-\mathbf{u}\|<\delta / 2\}$. Since these balls are disjoint their union fills up a region of volume $(\# U)(\delta / 2)^{m} V_{0}$ in the ball of radius $R+\delta / 2$. The latter ball has volume $(R+\delta / 2)^{m} V_{0}$. Hence $(\# U)(\delta / 2)^{m} \leq(R+\delta / 2)^{m}$ and our lemma follows. 
Lemma 3.7. Let $\Psi$ be a convex symmetric body in $\mathbb{R}^{r}$. By $\lambda \Psi$ we denote the convex body obtained by multiplying the points of $\Psi$ by $\lambda$. Then, for any $\lambda>1$, the set $\lambda \Psi$ can be covered by $(4+2 \lambda)^{r}$ translated copies of $\Psi$.

The proof of this lemma can be found in [6, Lemma 7.2]. However, we really need the following corollary.

COROLlary 3.8. Let $V$ be an r-dimensional normed real vector space with norm $\|\cdot\|$. Let $\varepsilon>0$. Then there is a finite set $E \subset V$ of unit vectors such that every $\mathbf{v} \in V$ can be written as $\mathbf{v}=\|\mathbf{v}\| \mathbf{e}+\mathbf{v}^{\prime}$ with $\mathbf{e} \in E$ and $\left\|\mathbf{v}^{\prime}\right\| \leq \varepsilon\|\mathbf{v}\|$. Moreover, $E$ can be chosen such that $\# E<(4+4 / \varepsilon)^{r}$.

Pr o of. Let $B$ be the unit ball with respect to $\|\cdot\|$. According to Lemma 3.7 the ball $B$ can be covered by $(4+4 / \varepsilon)^{r}$ translates of $(\varepsilon / 2) B$. Consider such a covering and let $\Delta$ be the subset of $(\varepsilon / 2)$-balls which have non-trivial intersection with the boundary of $B$. Clearly the balls in $\Delta$ give a covering of the boundary of $B$. For the set $E$ we take the unit vectors $\mathbf{c} /\|\mathbf{c}\|$ where c runs over the centers of the $(\varepsilon / 2)$-balls in $\Delta$.

Now let $\mathbf{v} \in \mathbb{R}^{r}$ be arbitrary. Let $\mathbf{c}$ be the center of the $(\varepsilon / 2)$-ball in $\Delta$ which contains $\mathbf{v} /\|\mathbf{v}\|$ and let $\mathbf{e}=\mathbf{c} /\|\mathbf{c}\|$. Notice that $\|\mathbf{c}-\mathbf{e}\|=|1-\|\mathbf{c}\|| \leq$ $\varepsilon / 2$. Hence,

$$
\left\|\frac{\mathbf{v}}{\|\mathbf{v}\|}-\mathbf{e}\right\| \leq\left\|\frac{\mathbf{v}}{\|\mathbf{v}\|}-\mathbf{c}\right\|+\|\mathbf{c}-\mathbf{e}\| \leq \varepsilon / 2+\varepsilon / 2=\varepsilon .
$$

Thus we find $\|\mathbf{v}-\| \mathbf{v}\|\mathbf{e}\| \leq \varepsilon\|\mathbf{v}\|, \mathbf{e} \in E$ and our corollary follows.

4. Proof of Theorem 1.1. Let $\Sigma$ be a subset of a normed vector space $V$ satisfying

1. $\left\|\mathbf{w}_{1}\right\| \leq \log 2+2\left\|\mathbf{w}_{2}-\mathbf{w}_{1}\right\|$ for any two distinct $\mathbf{w}_{1}, \mathbf{w}_{2} \in \Sigma$.

2. There exists $c_{1}$ such that $\left\|\mathbf{w}_{1}\right\| \leq c_{1}+(1 / \varrho)\left(\log 2+2\left\|\mathbf{w}_{2}-2 \varrho \mathbf{w}_{1}\right\|\right)$ for any two distinct $\mathbf{w}_{1}, \mathbf{w}_{2} \in \Sigma$ and any $\varrho \in \mathbb{N}$.

3. There exists $c_{0}>0$ such that $\max \left(\left\|\mathbf{w}_{1}-\mathbf{w}_{0}\right\|,\left\|\mathbf{w}_{2}-\mathbf{w}_{0}\right\|\right) \geq c_{0}$ for any three distinct $\mathbf{w}_{0}, \mathbf{w}_{1}, \mathbf{w}_{2} \in \Sigma$.

Proposition 4.1. Let $c_{2}=\max \left(2 \log 2, c_{1}+(\log 2) / 20\right)$. Then

$$
\# \Sigma \leq \frac{1}{2}\left(44+2 \frac{c_{2}}{c_{0}}\right)^{r+1}
$$

where $r$ is the dimension of $V$.

$\operatorname{Proof}$. Let $\varepsilon$ be a real number such that $0<\varepsilon<0.1$. Let $\mathbf{e}$ be a unit vector in $V$ and consider the cone

$$
C_{\mathbf{e}}=\left\{\mathbf{v} \in V: \mathbf{v}=\|\mathbf{v}\| \mathbf{e}+\mathbf{v}^{\prime},\left\|\mathbf{v}^{\prime}\right\| \leq \varepsilon\|\mathbf{v}\|\right\} .
$$


Let

$$
c_{3}(\varepsilon)=\frac{c_{2}}{1-10 \varepsilon} .
$$

We will show that for any two $\mathbf{w}_{1}, \mathbf{w}_{2} \in \Sigma \cap C_{\mathbf{e}}$ with $c_{3}(\varepsilon)<\left\|\mathbf{w}_{1}\right\| \leq\left\|\mathbf{w}_{2}\right\|$ we have

$$
(5 / 4)\left\|\mathbf{w}_{1}\right\| \leq\left\|\mathbf{w}_{2}\right\| \leq(1+4 / \varepsilon)\left\|\mathbf{w}_{1}\right\| .
$$

Suppose first $\mathbf{w}_{1}, \mathbf{w}_{2} \in \Sigma \cap C_{\mathbf{e}}$ and $\left\|\mathbf{w}_{1}\right\| \leq\left\|\mathbf{w}_{2}\right\|<(5 / 4)\left\|\mathbf{w}_{1}\right\|$. Write $\mathbf{w}_{i}=\left\|\mathbf{w}_{i}\right\| \mathbf{e}+\mathbf{w}_{i}^{\prime}$. Then, from the first inequality on $\Sigma$, we infer

$$
\begin{aligned}
\left\|\mathbf{w}_{1}\right\| & \leq \log 2+2\left\|\left(\left\|\mathbf{w}_{2}\right\|-\left\|\mathbf{w}_{1}\right\|\right) \mathbf{e}+\mathbf{w}_{2}^{\prime}-\mathbf{w}_{1}^{\prime}\right\| \\
& \leq \log 2+2\left(\left\|\mathbf{w}_{2}\right\|-\left\|\mathbf{w}_{1}\right\|\right)+2 \varepsilon\left(\left\|\mathbf{w}_{2}\right\|+\left\|\mathbf{w}_{1}\right\|\right) \\
& \leq \log 2+2(1 / 4)\left\|\mathbf{w}_{1}\right\|+2 \varepsilon(9 / 4)\left\|\mathbf{w}_{1}\right\| .
\end{aligned}
$$

We obtain

$$
\left\|\mathbf{w}_{1}\right\| \leq \frac{2 \log 2}{1-9 \varepsilon} \leq c_{3}(\varepsilon) .
$$

Suppose next that $\mathbf{w}_{1}, \mathbf{w}_{2} \in \Sigma \cap C_{\mathbf{e}}$ and $\left\|\mathbf{w}_{2}\right\|>(1+4 / \varepsilon)\left\|\mathbf{w}_{1}\right\|$. Choose $\varrho \in \mathbb{N}$ such that $\left\|\mathbf{w}_{2}\right\|=(2 \varrho+\delta)\left\|\mathbf{w}_{1}\right\|$ with $|\delta| \leq 1$. Notice that $\varrho \geq 2 / \varepsilon$. From the second inequality on $\Sigma$ it follows that

$$
\begin{aligned}
\left\|\mathbf{w}_{1}\right\| & \leq c_{1}+\frac{1}{\varrho}\left(\log 2+2\|\delta\| \mathbf{w}_{1}\left\|\mathbf{e}+\mathbf{w}_{2}^{\prime}-2 \varrho \mathbf{w}_{1}^{\prime}\right\|\right) \\
& \leq c_{1}+(\log 2) / 20+\frac{2}{\varrho}\left(\left\|\mathbf{w}_{1}\right\|+\varepsilon\left(\left\|\mathbf{w}_{2}\right\|+2 \varrho\left\|\mathbf{w}_{1}\right\|\right)\right) \\
& \leq c_{2}+\frac{2}{\varrho}\left\|\mathbf{w}_{1}\right\|+\varepsilon(8+4 / \varrho)\left\|\mathbf{w}_{1}\right\| \\
& \leq c_{2}+\varepsilon\left\|\mathbf{w}_{1}\right\|+9 \varepsilon\left\|\mathbf{w}_{1}\right\| .
\end{aligned}
$$

We get

$$
\left\|\mathbf{w}_{1}\right\| \leq \frac{c_{2}}{1-10 \varepsilon} \leq c_{3}(\varepsilon) .
$$

We now put the above considerations together. Let $N$ be the smallest integer such that $(5 / 4)^{N-1}>1+4 / \varepsilon$. Suppose $C_{\mathbf{e}}$ contains $N$ points $\mathbf{w}_{1}, \ldots, \mathbf{w}_{N}$ larger than $c_{3}(\varepsilon)$. Suppose they are ordered by size. Then, for each $i,\left\|\mathbf{w}_{i+1}\right\| /\left\|\mathbf{w}_{i}\right\| \geq 5 / 4$. This implies $\left\|\mathbf{w}_{N}\right\| /\left\|\mathbf{w}_{1}\right\|>(5 / 4)^{N-1}>1+4 / \varepsilon$, which is impossible by inequality (4). Hence any cone $C_{\mathbf{e}}$ contains at most $N-1$ elements from $\Sigma$ of norm $\geq c_{3}(\varepsilon)$. According to Lemma 3.8 the space $V$ can be covered by $(4+4 / \varepsilon)^{r}$ such cones and so the total number of points of $\Sigma$ larger than $c_{3}(\varepsilon)$ can be estimated by $(N-1)(4+4 / \varepsilon)^{r}$. Since $\varepsilon<0.1$ it is not hard to see that $N-1<2 / \varepsilon$. Hence the number of large points is bounded by $(2 / \varepsilon)(4+4 / \varepsilon)^{r}$.

It remains to count the elements of $\Sigma$ with norm at most $c_{3}(\varepsilon)$. By the third inequality on $\Sigma$ a ball of radius $c_{0}$ around a point of $\Sigma$ contains at 
most one other element from $\Sigma$. Consider a subset $\Sigma^{\prime}$ of $\Sigma$ such that a ball of radius $c_{0}$ around any point of $\Sigma^{\prime}$ contains no other point of $\Sigma^{\prime}$. We can do this in such a way that $|\Sigma| \leq 2\left|\Sigma^{\prime}\right|$. According to Lemma 3.6 the number of points in $\Sigma^{\prime}$ can be bounded from above by $\left(1+2 c_{3}(\varepsilon) / c_{0}\right)^{r}$. Thus we conclude

$$
|\Sigma| \leq \frac{2}{\varepsilon}\left(4+\frac{4}{\varepsilon}\right)^{r}+2\left(\frac{2 c_{3}(\varepsilon)}{c_{0}}+1\right)^{r} .
$$

Now we choose $\varepsilon$ such that $4 / \varepsilon=2 c_{3}(\varepsilon) / c_{0}$, i.e. $\varepsilon=\left(10+0.5 c_{2} / c_{0}\right)^{-1}$. Our proposition then follows immediately.

Proof of Theorem 1.1. By a specialisation argument as in [5] we may assume that $G \subset\left(\overline{\mathbb{Q}}^{*}\right)^{2}$. We now complete the line of argument started in Section 3. There we had the set $\mathcal{M}$. This set satisfies the conditions of Proposition 4.1 for the values $c_{0}=0.022522 \ldots, c_{1}=\log (6 \sqrt{3})=2.3410 \ldots$ Hence the cardinality of $\mathcal{M}$ is bounded by $\frac{1}{2} \cdot 256^{r+1}$. Since the number of solutions of $(\mathrm{M})$ is bounded by $2 \# \mathcal{M}$ our theorem follows.

5. Proof of Theorem 1.2. We first need a lemma

Lemma 5.1. Consider the equation $\lambda \alpha^{x}+\mu \beta^{x}=1$ in $x \in \mathbb{Z}$ where $\lambda, \mu, \alpha, \beta$ are as in the introduction and assumed to be algebraic numbers. Suppose we have the solutions $x=0, r, s, t$. Suppose that $t \geq 14 s$. Then

$$
s-8.4 r \leq \frac{9.1}{\log H(\alpha, \beta, 1)} \text {. }
$$

Proof. Application of Corollary 2.2 with $A=\lambda, B=\mu$ yields

$$
H(\lambda, \mu, 1) \leq 2 H(\alpha, \beta, 1)^{r} .
$$

Apply Lemma 2.3 with $A=\lambda \alpha^{s}, B=\mu \beta^{s}$ and $\varrho$ such that $t=2 s \varrho+\delta$, with $0 \leq \delta<2 s$. Note that $\varrho \geq 7$. We obtain

$$
\begin{aligned}
H\left(\lambda \alpha^{s}, \mu \beta^{s}, 1\right) & \leq 2^{1 / \varrho} c H\left(\alpha^{\delta} \lambda^{1-2 \varrho}, \beta^{\delta} \mu^{1-2 \varrho}\right)^{1 / \varrho} \\
& \leq 2^{1 / \varrho} c H(\alpha, \beta, 1)^{\delta / \varrho} H\left(\lambda^{-1}, \mu^{-1}, 1\right)^{2-1 / \varrho} .
\end{aligned}
$$

Notice that

$$
\begin{aligned}
H(\alpha, \beta, 1)^{s} & \leq H\left(\lambda^{-1}, \mu^{-1}, 1\right) H\left(\lambda \alpha^{s}, \mu \beta^{s}, 1\right) \\
& \leq 2^{1 / \varrho} c H(\alpha, \beta, 1)^{\delta / \varrho} H\left(\lambda^{-1}, \mu^{-1}, 1\right)^{3-1 / \varrho}
\end{aligned}
$$

and use $H\left(\lambda^{-1}, \mu^{-1}, 1\right) \leq H(\lambda, \mu, 1)^{2} \leq 4 H(\alpha, \beta, 1)^{2 r}$ to obtain

$$
H(\alpha, \beta, 1)^{s-\delta / \varrho} \leq 2^{1 / \varrho} c 2^{6-2 / \varrho} H(\alpha, \beta, 1)^{6 r}<64 c H(\alpha, \beta, 1)^{6 r} .
$$

Taking $\log$ 's and using $\log (64 c) \leq 6.5$ yields

$$
s-\delta / \varrho-6 r \leq 6.5 / \log H(\alpha, \beta, 1)
$$

from which our lemma is immediate via $\delta / \varrho \leq 2 s / 7$. 
Proof of Theorem 1.2. By Theorem 2 of [1] we may assume that $\alpha, \beta, \lambda, \nu \in \overline{\mathbb{Q}}$. Without loss of generality we can also assume that

$$
H(\alpha, \beta, 1) \leq H\left(\alpha^{-1}, \beta^{-1}, 1\right) .
$$

Let $q$ be the length of the shortest closed interval containing three solutions. Let $n, n+p, n+q$ be such three solutions. Application of Lemma 2.4 to the equation $\lambda \alpha^{n+p} X+\mu \beta^{n+p} Y=1$ yields

$$
H(\alpha, \beta, 1)^{q-p} H\left(\alpha^{-1}, \beta^{-1}, 1\right)^{p} \geq c_{4},
$$

where $c_{4}=1.0942711 \ldots$ Hence $H\left(\alpha^{-1}, \beta^{-1}, 1\right)^{q} \geq c_{4}$.

Define $\gamma=\log 8 / \log c_{4}$ and note that $\gamma<23.1$.

Now let $k<l<m<n$ be any four solutions. First of all application of Corollary 2.2 with $A=\lambda \alpha^{k}, B=\mu \beta^{k}$ yields

$$
H\left(\lambda \alpha^{k}, \mu \beta^{k}, 1\right) \leq 2 H(\alpha, \beta, 1)^{l-k} .
$$

In a similar way application of Corollary 2.2 with $A=\lambda \alpha^{n}, B=\mu \beta^{n}$ yields

$$
H\left(\lambda \alpha^{n}, \mu \beta^{n}, 1\right) \leq 2 H\left(\alpha^{-1}, \beta^{-1}, 1\right)^{n-m} .
$$

Application of Lemma 2.1 with $A=\alpha^{k-n}, B=\beta^{k-n}$ yields

$$
\begin{aligned}
H\left(\alpha^{k-n}, \beta^{k-n}, 1\right) & \leq 2 H\left(\lambda \alpha^{n}, \mu \beta^{n}, 1\right) H\left(\lambda \alpha^{n-k+l}, \mu \beta^{n-k+l}, 1\right) \\
& \leq 2 H\left(\lambda \alpha^{n}, \mu \beta^{n}, 1\right)^{2} H\left(\alpha^{l-k}, \beta^{l-k}, 1\right) .
\end{aligned}
$$

With $(6)$ and $H(\alpha, \beta, 1) \leq H\left(\alpha^{-1}, \beta^{-1}, 1\right)$ we get

$$
H\left(\alpha^{-1}, \beta^{-1}, 1\right)^{n-k} \leq 8 H\left(\alpha^{-1}, \beta^{-1}, 1\right)^{2(n-m)+l-k} .
$$

Using our lower bound $H\left(\alpha^{-1}, \beta^{-1}, 1\right) \geq c_{4}^{1 / q}$ we find that

$$
n-2 m+l \geq-\gamma q \text { hence } n-l-\gamma q \geq 2(m-l-\gamma q) \text {. }
$$

Denote the smallest solution by $n_{0}$ and the second smallest by $n_{1}$. Application of the inequality with $k=n_{0}, l=n_{1}$ yields

$$
n-n_{1}-\gamma q \geq 2\left(m-n_{1}-\gamma q\right)
$$

for any two solutions $m, n$ with $n_{1}<m<n$. We divide our solutions into three intervals,

- $I_{1}=\left[n_{0}, n_{1}+(0.9+\gamma) q[\right.$,

- $I_{2}=\left[n_{1}+(0.9+\gamma) q, n_{1}+(230+\gamma) q[\right.$,

- $I_{3}=\left[n_{1}+(230+\gamma) q, \infty[\right.$.

Since any interval of length $<q$ contains at most two solutions, the interval $I_{1}$ contains at most $1+2([\gamma+0.9]+1) \leq 49$ solutions. Because of (7) the interval $I_{2}$ contains at most 8 solutions.

We finally show that $I_{3}$ contains at most 4 solutions. Suppose $I_{3}$ contains 5 solutions, the largest being denoted by $N$, the smallest by $M$. Furthermore, we let $k$ be a solution such that there exists another solution $l$ such that 
$k<l<k+q$. Because of (7) we find $k<n_{1}+(1+\gamma) q$. Since there exists at least one closed interval of length $q$ containing three solutions such a $k$ exists and we may moreover assume that $k \geq n_{1}$. From (7) it follows that $\left(N-n_{1}-\gamma q\right) \geq 16\left(M-n_{1}-\gamma q\right)$. Since $k \geq n_{1}$ this implies $(N-$ $k-\gamma q) \geq 16(M-k-\gamma q)$ and since $N-k>M-k>229 q$ we get $N-k \geq(16-15 \gamma / 229)(M-k)>14(M-k)$. Application of Lemma 5.1 to the equation $\lambda \alpha^{k} \alpha^{x}+\mu \beta^{k} \beta^{x}=1$ with $r=l-k, s=M-k, t=N-k$ yields

$$
M-k-8.4(l-k) \leq \frac{9.1}{\log H(\alpha, \beta, 1)} .
$$

Using the lower bound $H(\alpha, \beta, 1) \geq c_{4}^{1 /(2 q)}$ and $l-k<q$ we get $M-k<211 q$, contradicting $M-k>229 q$.

So we conclude that $I_{3}$ contains at most 4 solutions, which leaves us with a total of at most $49+8+4=61$ solutions.

\section{References}

[1] F. Beukers and R. Tijdeman, On the multiplicities of binary complex recurrences, Compositio Math. 51 (1984), 193-213.

[2] F. Beukers and D. Zagier, Lower bounds for heights of points on hypersurfaces, Math. Z., to appear.

[3] J. H. Evertse, On equations in S-units and the Thue-Mahler equation, Invent. Math. 75 (1984), 561-584.

[4] H. P. Schlickewei, The multiplicity of binary recurrences, ibid., to appear.

[5] -, Equations $a x+b y=1$, Ann. of Math., to appear.

[6] H. P. Schlickewei and W. M. Schmidt, Linear equations in variables which lie in a multiplicative group, to appear.

[7] H. P. Schlickewei and E. Wirsing, Lower bounds for the heights of solutions of linear equations, Invent. Math., to appear.

Department of Mathematics

University of Utrecht

Budapestlaan 6

P.O. Box 80010

3508 TA Utrecht, The Netherlands

E-mail: beukers@math.ruu.nl
Abteilung für Mathematik II

Universität Ulm

Helmholtzstr. 18 89069 Ulm, Germany

E-mail: hps@mathematik.uni-ulm.de 